\title{
Open
}

\section{Prevention of vaginal SHIV transmission in macaques by a live recombinant Lactobacillus}

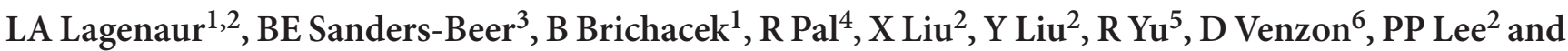 \\ DH Hamer ${ }^{1}$
}

Most human immunodeficiency virus (HIV) transmissions in women occur through the cervicovaginal mucosa, which is coated by a bacterial biofilm including Lactobacillus. This commensal bacterium has a role in maintaining a healthy mucosa and can be genetically engineered to produce antiviral peptides. Here, we report a $63 \%$ reduction in transmission of a chimeric simian/HIV (SHIV $\mathrm{SF162P3}_{3}$ ) after repeated vaginal challenges of macaques treated with Lactobacillus jensenii expressing the HIV-1 entry inhibitor cyanovirin-N. Furthermore, peak viral loads in colonized macaques with breakthrough infection were reduced sixfold. Colonization and prolonged antiviral protein secretion by the genetically engineered lactobacilli did not cause any increase in proinflammatory markers. These findings lay the foundation for an accessible and durable approach to reduce heterosexual transmission of HIV in women, which is coitally independent, inexpensive, and enhances the natural protective effects of the vaginal microflora.

\section{INTRODUCTION}

Halting the global human immunodeficiency virus (HIV)/AIDS (acquired immunodeficiency syndrome) epidemic requires interventions that have practical and achievable goals in the developing world setting. Topical prevention strategies designed to interdict infection at the mucosal surface represent one approach. Early prevention trials examined nonspecific microbicides, such as detergents and polyanions (e.g., Nonoxynol-9 and cellulose sulfate), which were wholly ineffective or in some cases may have increased the risk of HIV acquisition because of the inflammatory and viral-enhancing properties of the compounds used. ${ }^{1-7}$ These clinical trials also revealed that coitally dependent products, such as Carraguard, were used less frequently than women reported, ${ }^{8}$ highlighting the difficulty of achieving the level of adherence required for efficacy of such microbicides. Recent results from the CAPRISA 004 trial provided a much-needed proof of concept that a topical agent, the specific antiretroviral drug tenofovir delivered as a gel, can prevent the acquisition of HIV in women. ${ }^{9}$ However, the efficacy of this approach was modest (39\%), probably in large part because it is a coitally dependent regimen and the gel must be applied within $12 \mathrm{~h}$ both before and after sexual intercourse for maximum efficacy. It was concluded from the trial results that the main goals are to improve adherence and to develop coitally independent microbicide strategies.

In many cultural settings, women need a product that can be used covertly without obtaining the permission of their sexual partner. In addition, the cost of HIV prevention must be affordable to the developing world. Thus, there is still a need for products that block HIV transmission, are safe and easy to use, and are coitally independent, discreet, and cost effective.

The majority of viral transmissions in women occur during unprotected vaginal intercourse on the mucosal surfaces of the endocervix, cervix, and vagina. In macaques ${ }^{10}$ and human vaginal explant tissues, ${ }^{11,12}$ Langerhans cells and intraepithelial CD $4+$ T cells are the targets of HIV-1 viral entry and initial replication. ${ }^{12}$ Mucosal surfaces of the lower female reproductive tract are normally coated with a biofilm of commensal bacteria including lactobacilli, which are an important component of the vaginal microbiome and provide the first line of defense against invading pathogens. ${ }^{13}$ Lactobacilli acidify the vagina by producing lactic acid ${ }^{14}$ and may reduce the risk of acquiring HIV-1 and other sexually transmitted infections. ${ }^{15,16}$

One practical and cost-effective approach to protect the vaginal epithelium is to colonize it with a recombinant Lactobacillus expressing an antiviral protein, thus transforming the vaginal

${ }^{1}$ Gene Structure and Regulation Section, Vaccine Branch, National Cancer Institute, National Institutes of Health, Bethesda, Maryland, USA. 2Osel, Mountain View, California, USA. ${ }^{3}$ BIOQUAL, Rockville, Maryland, USA. ${ }^{4}$ Advanced BioScience Laboratories, Rockville, Maryland, USA. ${ }^{5}$ Cepheid, Sunnyvale, California, USA. ${ }^{6}$ Biostatistics and Data Management Section, National Cancer Institute, National Institutes of Health, Rockville, Maryland, USA. Correspondence: LA Lagenaur (llagenaur@mail.nih.gov) 
microflora into a "live" bioshield. ${ }^{17}$ This is a novel approach to preventing the transmission of HIV, and eventually other mucosal pathogens, and thereby represents a platform approach to intervention of disease transmission that is both discreet to the user and partner. Importantly, it is also inexpensive to manufacture.

Cyanovirin-N (CV-N), a protein originally isolated from the cyanobacterium, Nostoc elipsosporum, was selected as a model HIV inhibitor for this approach. CV-N has broad cross-clade potency against HIV ${ }^{18,19}$ including primary isolates. ${ }^{20,21}$ Previous studies have shown that the CV-N protein, formulated in a gel, protected macaques from vaginal and rectal challenge. $^{22,23}$

We previously described a Lactobacillus strain, Lactobacillus jensenii 1153-1666, which is genetically engineered to secrete an amino-terminally modified CV-N protein (P51G). ${ }^{24}$ The CV-N protein isolated from the culture media of this strain has potent anti-HIV activity against CCR5- and CXCR4-tropic viruses in vitro, whereas control media from the parental strain, $L$. jensenii 1153 OSEL 175, are inactive. ${ }^{24}$ Compared with the parental strain, $L$. jensenii 1153-1666 displays no loss of fitness, grows to the same levels, and produces similar levels of lactic acid and hydrogen peroxide. ${ }^{24}$ Although L. jensenii 1153-1666 persisted in the estrogenized CD1 mouse model, ${ }^{24}$ mice do not support Lactobacillus colonization as they have significantly different vaginal biology from primates. Therefore, to advance this platform technology, a nonhuman primate model was developed.

The rhesus macaque model is commonly used for microbicide and vaccine studies, but has not previously been used for testing live microbicides; therefore, we performed extensive characterization to determine whether rhesus macaques were suitable as a model. We found that Chinese- and Indian-origin rhesus macaques typically harbor abundant endogenous vaginal Lactobacillus johnsonii as their predominant species, and, like humans, generally have only one vaginal Lactobacillus strain at a time. ${ }^{25}$ We examined the DNA fingerprints and biochemical profiles of $L$. johnsonii strains and found that most are strong $\mathrm{H}_{2} \mathrm{O}_{2}$ producers and are similar to the human strain Lactobacillus gasseri. ${ }^{25}$ We further characterized changes in the vaginal microbiome during menses. Unlike humans, the endogenous Lactobacillus flora of the macaque varies more throughout the menstrual cycle, with the lowest levels found at or around menstruation. ${ }^{25,26}$ Vaginal $\mathrm{pH}$ in macaques is also higher than in humans with the average value $\sim 6.3$ among the animals in this study. Higher vaginal $\mathrm{pH}$ is also found in women with bacterial vaginosis, which predisposes them to increased risk of HIV infection. ${ }^{16}$ Thus, it has recently been suggested that the macaque may be a useful model for HIV protection studies because they resemble women with bacterial vaginosis. ${ }^{27}$ Displacement of endogenous lactobacilli, followed by longterm colonization of rhesus macaques with human $L$. jensenii 1153 strain has been achieved, making this animal model useful for preclinical evaluation of live Lactobacillus-based antiviral approaches. ${ }^{25}$

Here, we used the macaque model to test colonization of recombinant lactobacilli, CV-N protein expression, and efficacy of the recombinant $L$. jensenii 1153-1666 in a repeated vaginal simian/HIV (SHIV) challenge model, and to demonstrate that colonized animals show a $63 \%$ reduction in acquisition of the virus. We also examined vaginal $\mathrm{pH}$, histology, and biomarkers of inflammation to assess the safety of this approach.

\section{RESULTS}

\section{Colonization and protein expression of recombinant Lactobacillus in macaques}

First, we examined the duration of colonization and in situ protein expression using L. jensenii 1153-1666 in macaques. Vaginal inoculation of two macaques, M1 and M2, with live L. jensenii 1153-1666 delivered in hydroxyethylcellulose (HEC) on five successive days led to sustained colonization and secretion of full-length CV-N that was detected in cervical-vaginal lavage $(\mathrm{CVL})$ as early as $24 \mathrm{~h}$ after inoculation, and persisted at 3 and at 6 weeks after inoculation (Figure 1). Decreases in CV-N levels in $\mathrm{CVL}$, as seen in animal M1 at week 3, appear to be due to lower levels of Lactobacillus colonization during menses. ${ }^{25} \mathrm{CV}-\mathrm{N}$ in CVLs collected from macaques was measured at a concentration of $83-160 \mathrm{ng} \mathrm{ml}^{-1}$.

Histology and immunohistochemistry were performed on vaginal biopsies collected from control macaques or macaques colonized with $L$. jensenii 1153-1666. Colonization

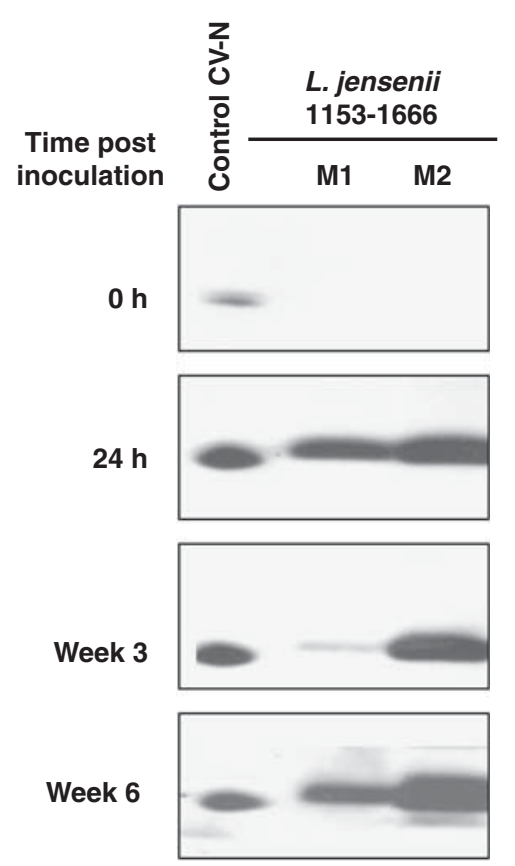

Figure 1 Expression and detection of CV-N in situ in the macaque vagina. L. jensenii 1153-1666 was used to colonize macaques for a 5 -day period. After this dosing regimen, cervical-vaginal lavages (CVLs) were taken at $24 \mathrm{~h}$, and at 3 and 6 weeks. Samples of each CVL were immunoprecipitated and then visualized by western blot. Expression of CV-N was constitutive for up to 6 weeks post inoculation. At week 3 , monkey 1 (M1) had a very low but detectable CV-N protein band, likely caused by reduced levels of $\mathrm{CV}$-N-producing lactobacilli due to menstruation. L. jensenii 1153-1666, measured in CVL, grew on vaginal epithelial cells in vivo at concentrations up to $7 \times 10^{6}$ c.f.u. and yielded CV-N at 83-160 $\mathrm{ng} \mathrm{ml}^{-1}$. CV-N, cyanovirin-N. 
by the recombinant Lactobacillus did not lead to observable inflammation or disruption of squamous epithelial cell integrity as indicated by examination of vaginal histology sections from $L$. jensenii 1153-1666-colonized macaques compared with controls (Figure 2a). To detect CV-N in situ, biopsies were incubated with control serum or anti-CV-N serum. The CV-Nspecific serum revealed co-localization of the CV-N protein with Lactobacillus bacteria on the vaginal mucosa and in the vaginal lumen (Figure 2b).

\section{Examination of mucosal markers of inflammation, vaginal pH, and immunogenicity}

To determine the effects of the recombinant Lactobacillus on the vaginal environment, we performed sequential measurements of proinflammatory cytokines and $\mathrm{pH}$ in CVL of animals in the absence of detectable Lactobacillus, in the presence of spontaneously occurring endogenous L. johnsonii, and in the presence of introduced recombinant $L$. jensenii 1153-1666. The cytokine measurements focused on four proinflammatory mediator cytokines that are often used as biomarkers of inflammation and indicators of mucosal barrier function: interleukin (IL)$1 \beta$, IL-1RA, IL-6, and IL-8. ${ }^{28,29}$ As shown in Figure 3a-d, there was a high variation from animal to animal in the measured values of these cytokines, but no consistent or statistically significant differences as a function of bacterial colonization status. The average vaginal $\mathrm{pH}$ value of the macaques used in this study, as determined by seven sequential measurements, was $6.3 \pm 0.78$ (mean \pm s.e.m.). Comparison of uncolonized animals with those harboring endogenous L. johnsonii or introduced L. jensenii 1153-666 did not reveal any significant variations (Figure 3e). In addition, macaques that were repeatedly exposed to L. jensenii 1153-1666 had no detectable IgG, IgA, or IgM response to either CV-N or killed Lactobacillus measured in CVL, whereas macaques immunized through the intramuscular route with $100 \mu \mathrm{g} \mathrm{ml}^{-1}$ purified CV-N delivered in adjuvant exhibited a robust mucosal and systemic immune response to $\mathrm{CV}-\mathrm{N}$ (data not shown).

\section{Inhibition of HIV-1 infection in an EpiVaginal tissue model}

The MatTek EpiVaginal Tissue Model (MatTek, Ashland, MA) was used to assess the activity of $L$. jensenii 1153 (parental strain) vs. L. jensenii 1153-1666 (producing CV-N). Infection was monitored by $\mathrm{p} 24$ release at days 3, 5, and 8 after infection. It was shown that co-cultivation of the tissue model with the $L$. jensenii 1153 parental strain reduced infection by $23 \%$, compared with a control with no added bacteria $(P=0.1)$, whereas co-cultivation with CV-N-producing $L$. jensenii 1153-1666 inhibited infection by $72 \%(P<0.008)$ (Figure 4$)$.

\section{SHIV challenge protocol and results}

To determine the efficacy of $L$. jensenii 1153-1666 live prevention strategy in the macaque model, we designed a repetitive vaginal challenge protocol using the well-characterized CCR5-tropic virus $\mathrm{SHIV}_{\mathrm{SF162P3}}$. The repeated challenge model was chosen because it more closely resembles human infection than does a single challenge using either a very high viral dose or pretreatment with DepoProvera. The study protocol is shown in Figure 5a. In all, 12 menstrually cycling adult female macaques were given vaginal azithromycin suppositories to reduce endogenous Lactobacillus flora. They were then colonized with L. jensenii 1153-1666 for 5 days initially, and again at 48 and $24 \mathrm{~h}$ before each of 6-weekly challenges. Each challenge was

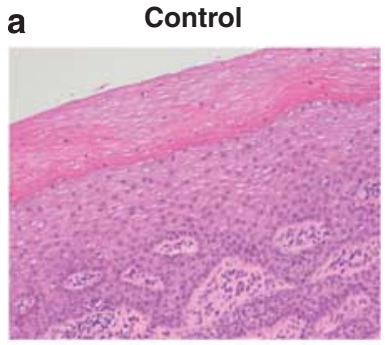

b

LB-treated
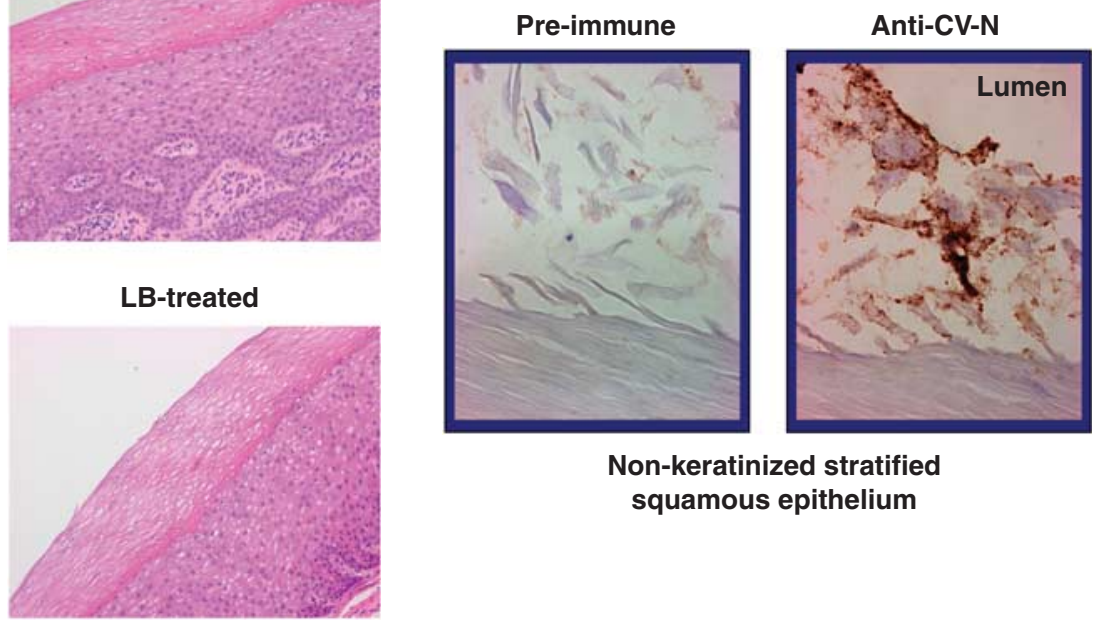

Figure 2 Vaginal histology and CV-N localization in the vagina. (a) Histology of vaginal biopsies. A biopsy from a macaque colonized with $L$. jensenii 1153-1666 for 1 month (bottom) and one from a control macaque (top) were compared. Formalin-fixed sections were stained with hematoxylin and eosin and examined microscopically. There was no evidence of loss of epithelial cell integrity and no leukocytic infiltration noted. Two representative biopsies are shown at $\times 20$ magnification. (b) Immunohistochemical analysis of CV-N in a vaginal biopsy. The biopsy on the left is reacted with pre-immune serum and shows no staining. In the biopsy on the right, CV-N staining co-localized with the Lactobacillus bacteria on the vaginal mucosal surface and in the vaginal lumen. CV-N, cyanovirin-N. 

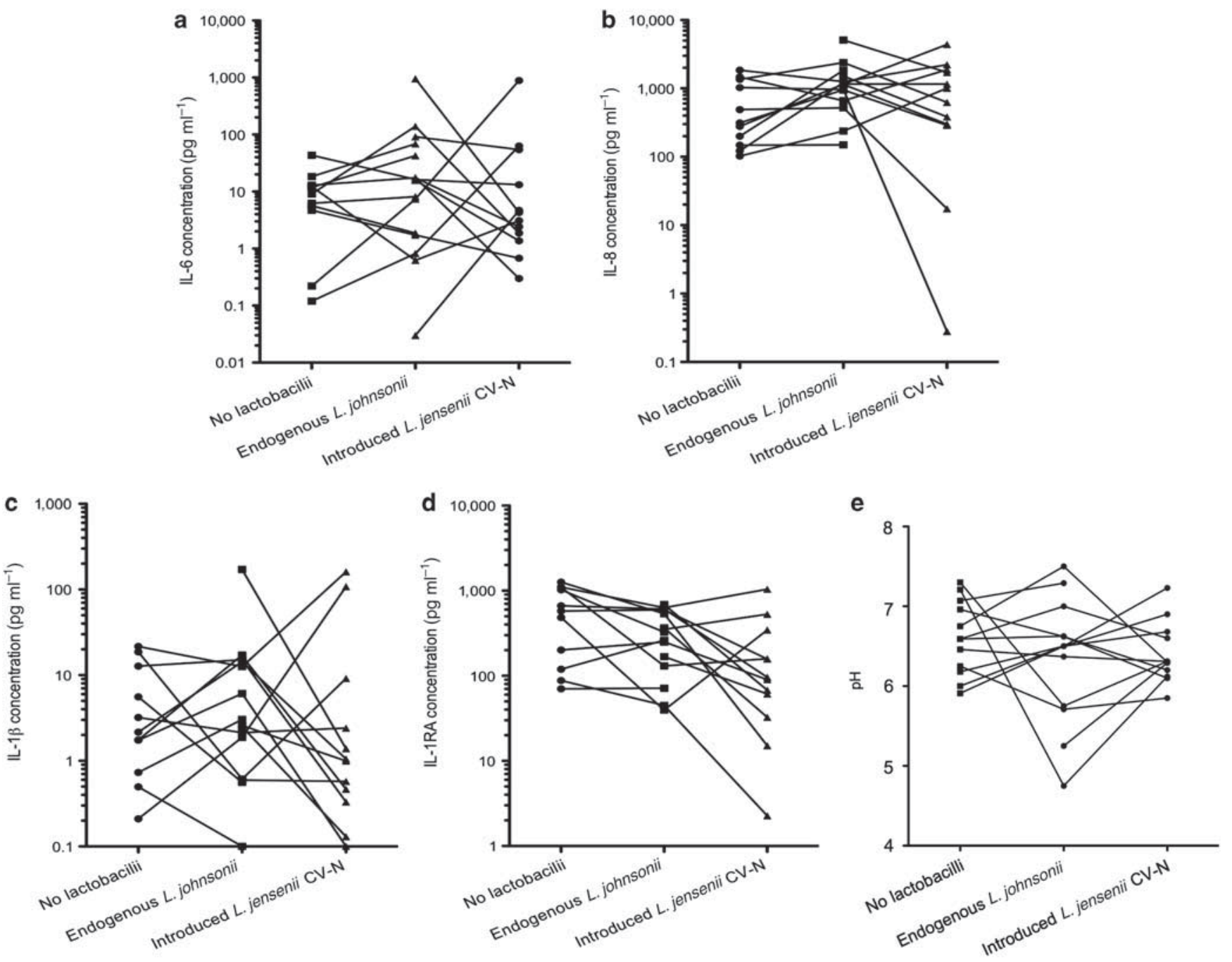

Figure 3 Measurements of biomarkers of inflammation in CVL and pH. The levels of (a) IL-6, (b) IL-8, (c) IL-1 $\beta$, and (d) IL-1RA, were determined in CVLs of 16 macaques with no lactobacilli, or colonized with either endogenous strains of $L$. johnsonii or with introduced $L$. jensenii $1153-1666$. Comparisons were made within the same macaque. (e) Vaginal $\mathrm{pH}$ values were determined from 12 macaques at multiple time points using a portable Skin $\mathrm{pH}$ meter. Comparisons were made for the same macaque with no lactobacilli or colonized either with endogenous strains of $L$. johnsonii or with L. jensenii 1153-1666. CVL, cervical vaginal lavage; CV-N, cyanovirin-N; IL, interleukin; RA, receptor antagonist.

performed a minimum of $24 \mathrm{~h}$ after bacterial inoculation, a period sufficient for CV-N protein accumulation to occur (Figure 1a) and to demonstrate the potential of coital independence. The challenge contained 300 median $\operatorname{TCID}_{50}$ (tissue culture infectious doses) of $\mathrm{SHIV}_{\mathrm{SF} 162 \mathrm{P} 3}$; this amount of virus was previously determined to infect $\sim 1$ of 3 animals after each challenge. We confirmed the presence and quantity of $L$. jensenii 1153-1666 by culture and the expression of CV-N by analysis of recovered L. jensenii 1153-1666 (Table 1).

The results of the challenge study are shown as a Kaplan-Meier analysis (Figure 5b). In all, 12 control macaques were infected at a rate of $35.4 \%$ after each vaginal $\mathrm{SHIV}_{\mathrm{SF} 162 \mathrm{P} 3}$ challenge; 31 challenges were required to infect 11 controls $(11 / 31=35.4 \%)$, with 1 control macaque remaining uninfected after 6 challenges. By contrast, 12 macaques treated with $L$. jensenii 1153-1666 (LB-CV-N) were infected significantly less frequently: 61 challenges were required to infect $8 \mathrm{LB}-\mathrm{CV}-\mathrm{N}$-treated macaques
$(8 / 61=13.1 \%)$, with 4 macaques remaining uninfected after 6 challenges. Thus, this live prevention strategy reduced the infection rate by $62.9 \%(P=0.0039)$.

The data were further analyzed by separately considering the three subgroups of the 12 control animals that received no exogenous Lactobacillus: those that received no treatment of any type $(n=4)$, HEC only $(n=4)$, or azithromycin + HEC $(n=4)$. Figure $5 \mathrm{c}$ shows that each of these subgroups has a greater infection rate than experimental animals, and this difference was significant for the nontreatment group $(P=0.008)$ and for the HEC-only group $(P=0.002)$.

Unexpectedly, we also observed that peak SHIV RNA plasma viral levels in macaques with breakthrough infection were sixfold lower in experimental animals that had been colonized with L. jensenii 1153-1666 compared with infected noncolonized control animals (Figure 6). Despite variability in the peak viral loads, this difference was significant $(P=0.029)$. 


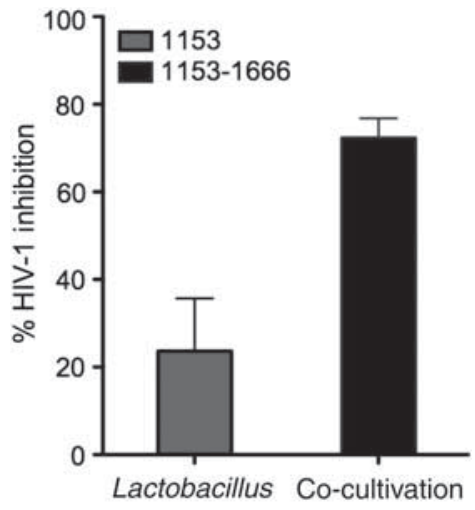

Figure 4 Inhibition of HIV-1 infection in a MatTek EpiVaginal tissue model. Initial in vitro experiments were performed with HIV-1 ${ }_{\text {SF162 }}$ infection of the MatTek EpiVaginal Tissue Model VLC-100 FT. We show that co-cultivation of the tissue model with the $L$. jensenii 1153 parental strain reduced infection by $23 \pm 12.1 \%$ (mean \pm s.e.m.), compared with a control with no added bacteria, whereas co-cultivation with CV-Nproducing $L$. jensenii $1153-1666$ inhibited infection by $72 \pm 4.5 \%$ (mean \pm s.e.m.). Supernatants were harvested for p24 analysis and HIV-1 infection was determined by p24 ELISA (Perkin-Elmer). CV-N, cyanovirin-N; HIV-1, human immunodeficiency virus-1.

The serological response to SHIV was evaluated by testing all plasma samples for seroconversion and quantifying antibody titers to $\mathrm{p} 27$, a simian immunodeficiency virus (SIV) core antigen. All macaques with detectable plasma viral load also generated antibodies to SIV-specific antigens with a prominent p27 protein band in western blots and positive titers to p 27 by enzyme-linked immunosorbent assay (ELISA), whereas all protected animals had no detectable SIV-specific antibodies by either western blot or ELISA (data not shown).

In addition, we measured TRIM5 $\alpha$ alleles for 20 of 24 macaques used in the study, including all 12 Lactobacillusinoculated animals and 8 control animals (Supplementary Table 1 online). We found no correlation between TRIM $5 \alpha-$ resistant or TRIM5 $\alpha$-sensitive phenotype and rate of infection or viral load.

\section{DISCUSSION}

This study examined a live recombinant Lactobacillus producing an HIV-entry inhibitor on the cervical/vaginal mucosa as a means to prevent transmission of $\mathrm{SHIV}_{\mathrm{SF} 162 \mathrm{P} 3}$ to macaques. Genetically engineered lactobacilli, which are capable of forming a colonizing biofilm on the cervical/vaginal mucosa, can provide a continuous delivery system for antiviral proteins. Furthermore, they provide beneficial protective effects of a normal vaginal microbiome, which helps to control various vaginal infections through multiple mechanisms including $\mathrm{H}_{2} \mathrm{O}_{2}$ and lactic acid production. ${ }^{13-16}$

Given the multifactorial nature of this approach, this study was designed to evaluate the combined, natural, and engineered antiviral properties of $L$. jensenii 1153-1666 rather than to focus only on antiviral protein expression. Control animals were not experimentally colonized with wild-type lactobacilli, but two control subgroups did receive either HEC placebo gel, which was used as a carrier for the L. jensenii 1153-1666, or HEC plus azithromycin, an antibiotic that was administered to reduce endogenous flora. There was a statistically significant difference in protection between $L$. jensenii 1153-1666-colonized animals and the control group as whole and between $L$. jensenii 1153-1666-colonized animals and the control nontreated or HEC-only subgroups. However, the number of animals in the individual subgroups was too small for a definitive subgroup comparison.

Now that the efficacy of $L$. jensenii 1153-1666 has been established, further experiments are required to differentiate the effects of the host organism as compared with the produced protein. Lactobacillus is associated with good vaginal health, and may have some degree of indigenous protective activity against various pathogens including HIV. Initial in vitro experiments using HIV infection of a human vaginal tissue model showed that co-cultivation with the L. jensenii 1153 parental strain reduced infection by $23 \%$ (but did not reach statistical significance; $P=0.10$ ) compared with a control with no added bacteria, whereas co-cultivation with CV-N-producing $L$. jensenii 11531666 inhibited infection by $72 \%(P<0.008)$. By contrast, in a MAGI cell-attachment assay, total conditioned media from the parental strain showed no activity compared with strong inhibition by material from CV-N-producing cells, suggesting that whatever inhibition is due to the innate activity of Lactobacillus occurs through a mechanism distinct from the fusion-inhibiting activity of CV-N (data not shown).

An essential criterion for a successful microbicide is that it should not cause inflammation, which is well known to increase susceptibility to HIV infection. ${ }^{16,30-32}$ In our study, we found no evidence for $L$. jensenii 1153-1666-induced inflammation either by microscopic examination of vaginal biopsy samples or by measurement of multiple proinflammatory mediator cytokines. In addition, CV-N did not appear to be immunogenic.

In many women, lactobacilli are the dominant flora and contribute to the average $\mathrm{pH}$ value of $\sim 4$ through lactic acid production. ${ }^{14}$ The $\mathrm{pH}$ of the vagina may also influence HIV transmission, with low $\mathrm{pH}$ values acting in a protective manner. However, it is important to note that HIV-1 is introduced in semen; thus, vaginal $\mathrm{pH}$ can transiently fluctuate to neutral or even basic $\mathrm{pH}$ for several hours after coitus. ${ }^{33}$ The CV-N protein is active in a range from $\mathrm{pH} 4$ to $8.2 .{ }^{17}$

In this model, we show that macaques have a more dynamic and complex flora, which is more similar to women with bacterial vaginosis. Thus, the macaque study may underestimate the potential efficacy of recombinant lactobacilli in humans, in whom the bacteria will have a more dominant role. Importantly, a phase 2a study has shown that $61-78 \%$ of women with bacterial vaginosis can be re-colonized with an introduced Lactobacillus crispatus strain. ${ }^{34}$

The CV-N protein was used in this study because it has broad activity against a wide range of HIV-1 isolates, it is stable in the vaginal environment, and it is potent at achievable concentrations. Huskens et al. ${ }^{35}$ have questioned the safety of CV-N as a topical microbicide because they observed mitogenic activity and enhanced expression of T-cell activation in peripheral 
a $\downarrow$ Challenges $300 \mathrm{TCID}_{50}$

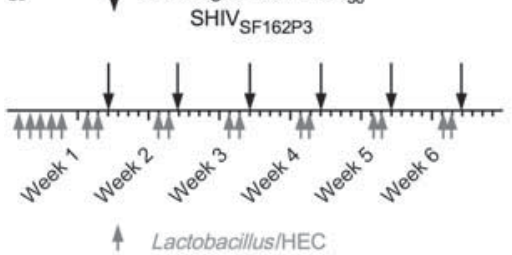

\begin{tabular}{|l|c|c|c|c|}
\hline Macaque & Azithromycin & $\begin{array}{c}\text { L. jensenii } \\
1153-1666\end{array}$ & $\begin{array}{c}2.7 \% \\
\text { HEC }\end{array}$ & $\begin{array}{c}\text { No } \\
\text { treatment }\end{array}$ \\
\hline Experimental & 12 & 12 & 12 & \\
\hline Control & 4 & & 4 & \\
\hline Control & & & 4 & \\
\hline Control & & & & 4 \\
\hline
\end{tabular}
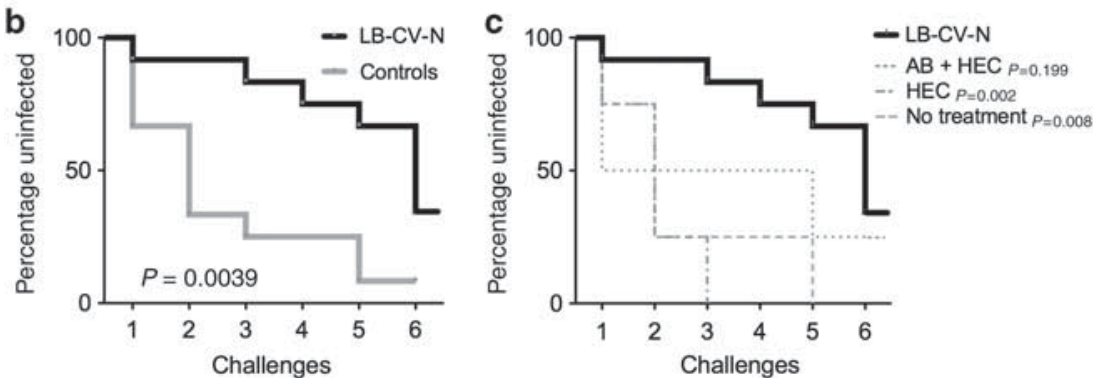

Figure 5 L. jensenii 1153-1666 protected rhesus macaques against repeated vaginal challenge by SHIV $\mathrm{SF}_{\text {SF2P3. }}$ (a) Study protocol. Adult female rhesus macaques $(n=12)$ were treated with the antibiotic azithromycin vaginally 1 week before the study. Animals were inoculated with $L$. jensenii 1153-1666 in 2.7\% HEC for a 5-day period, and then again for 2 days before each challenge. A total of 12 control macaques were used. Control macaques received the following: azithromycin treatment (as described above), followed by $2.7 \% \mathrm{HEC}$ (as described) $(n=4), 2.7 \% \mathrm{HEC}$ (as described) $(n=4)$ or no treatment $(n=4)$. Macaques were challenged once weekly up to six challenges. (b) Kaplan-Meier analysis of time to detectable SHIV $_{\mathrm{SF} 162 \mathrm{P} 3}$ infection. On the left, all controls were combined; the difference in protection was highly significant $(P=0.0039)$. (c) On the right, each subgroup within the controls was separately analyzed; the difference was significant for the nontreatment subgroup $(P=0.008)$ and the HEC-only subgroup $(P=0.002)$. HEC, hydroxyethylcellulose; SHIV, simian/human immunodeficiency virus.

Table 1 L. jensenii 1153-1666 and CV-N protein detection in L. jensenii 1153-1666-colonized macaques during the course of the challenge study

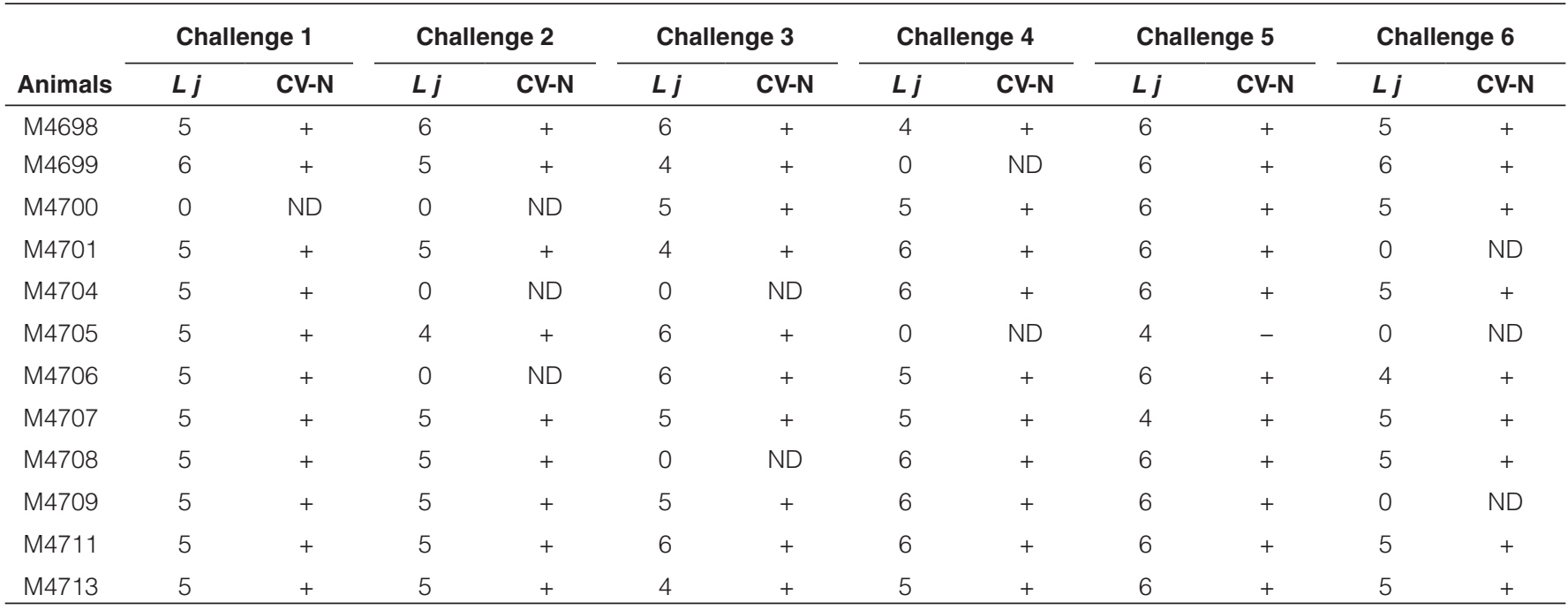

Abbreviations: $L j$, Lactobacillus jensenii 1153-1666; 6, $10^{6}$ colony-forming units (c.f.u.) per swab; $5,10^{5}$ c.f.u. per swab; 4, 104 c.f.u. per swab; 0, no Lactobacillus detected; ND, not determined.

blood mononuclear cells exposed to certain preparations of the protein. However, the clinical relevance of the study by Huskens et al. is unclear as it is not known whether proteins used in their analysis were monomeric (P51G) ${ }^{36}$ or dimeric (P51) or whether they were contaminated with endotoxin. By contrast, a study by Buffa et al. ${ }^{20}$ found low levels of T-cell proliferation only during prolonged exposure to extremely high CV-N concentrations $(360,000 \mathrm{nM})$ with no tissue toxicity. We observed that expression of the low 1-2 $\mu \mathrm{g} \mathrm{ml}^{-1}(92-183 \mathrm{~nm})$ levels of monomeric CV-N (P51G) produced by L. jensenii 1153-1666 did not enhance the expression of typical markers associated with mucosal barrier function or inflammation. Although additional biomarkers evaluation is required to determine any toxicity that may be seen with long-term use, these preliminary studies suggest that this low level is safe.

With the recent characterization of early HIV-1 infection events and findings that a majority of HIV transmissions involve a single founder virus, ${ }^{37}$ there has been a re-examination of 


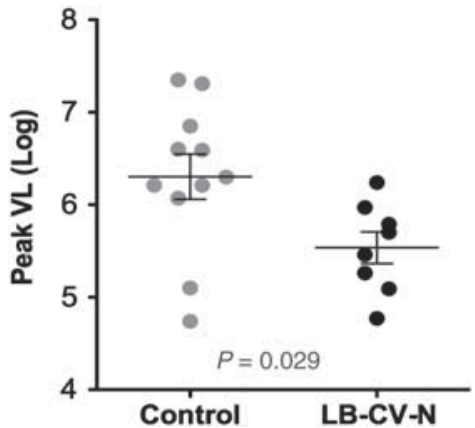

Figure 6 Comparison of peak viral loads in L. jensenii 1153-1666 colonized (LB-CV-N) and control animals with breakthrough infection. Average $\log _{10}$ peak plasma viral levels were $5.5 \pm 0.17$ (mean \pm s.e.m.) for animals colonized with $L$. jensenii $1153-1666$ vs. $6.3 \pm 0.24$ for control animals $(P=0.029)$. CV-N, cyanovirin-N.

SHIV and SIV models in an attempt to mimic the physiological dosage seen in natural infection. This has led to the use of lower doses with repeated challenges, requiring more prolonged studies but being more physiologically relevant than high-dose models. For example, low-dose rectal inoculations of macaques with SIV were shown to more closely recapitulate human mucosal infection by HIV- $1^{38}$ in terms of the number of viral variants that were transmitted. Repeated challenge approaches also increase the statistical power achieved. ${ }^{26,39}$ Perhaps most importantly, lower-challenge doses may be responsive to clinically achievable doses of microbicides; protection against high doses typically requires extraordinarily high and potentially toxic doses of the tested compounds. . $2,23,40-42$ For example, when tested in a cynomolgus macaque model, $0.5-2 \% \mathrm{CV}-\mathrm{N}$, equivalent to $5-20 \mathrm{mg} \mathrm{ml}^{-1}$ (454 $\left.\mu \mathrm{M}-1.8 \mathrm{~mm}\right)$ was required to inhibit $83 \%$ of infections in a very stringent vaginal challenge of 5,000 $\mathrm{TCID}_{50}$ on the epithelium that was thinned with medroxyprogesterone. ${ }^{22}$ Such early studies led to the belief that vast excesses of microbicide were required to provide efficacy and led to concerns about toxicity of the products used at such high levels.

These factors led us to the selection of a repeated low-dose vaginal challenge model for testing $L$. jensenii 1153-1666. The SHIV $_{\text {SF162P3 }}$ stock was diluted to a level that infects untreated macaques at $\sim 30 \%$ per challenge. This infection rate is still higher than that for women infected heterosexually, thus providing a stringent test of protection, yet more closely mimics natural infection than the use of a single high dose.

The reduction in viral load in macaques receiving $L$. jensenii 1153-1666 was an unexpected finding. It has been hypothesized that small founder populations of virus undergo local expansion before systemic dissemination. ${ }^{43}$ It is possible that these initial foci of infection are still accessible to the mucosally produced CV-N, providing some level of viral control after the initial infection event.

Certain virus strains, such as $\mathrm{SIV}_{\mathrm{E} 660}$, are restricted by TRIM5 $\alpha$ alleles. We found no correlation between susceptibility related to TRIM alleles. Restrictive MHC alleles, such as MamuA01, are rare in Chinese-origin macaques, but are more common in Indian-origin macaques. ${ }^{4,45}$
In this study, macaques were repeatedly colonized with L. jensenii 1153-1666 at least $24 \mathrm{~h}$ before each viral challenge. However, because this Lactobacillus strain was shown to colonize and secrete CV-N for at least 6 weeks after administration, it likely has the capacity to provide considerably more durable protection with relatively infrequent re-colonization. The ability of the recombinant Lactobacillus to continually colonize also suggests that our introduction of a single gene into the bacterial chromosome did not have a deleterious effect on its growth and survival in vivo. Importantly, because healthy women have 10-fold higher levels of Lactobacillus colonization in the cervical and vaginal mucosa than do female rhesus macaques, this approach may actually lead to even greater protection in humans than observed in this model.

The live L. jensenii 1153-1666 reduced the rate of $\mathrm{SHIV}_{\mathrm{SF} 162 \mathrm{P} 3}$ acquisition by $63 \%$ in the macaque model. Statistical modeling predicts that a reduction in the rate of HIV acquisition by $50 \%$ using either a partially effective vaccine or microbicide could have a significant impact on the global HIV epidemic. ${ }^{46,47}$ Our data suggest that this approach to HIV prevention is effective and safe. It is also highly flexible as the modular nature of the Lactobacillus expression system readily allows the expression of other HIV inhibitor proteins, such as the CCR5 inhibitor C1C5 RANTES, ${ }^{48}$ or even the production of multiple inhibitors. Furthermore, because lactobacilli are inexpensive to ferment and manufacture, this approach is eminently suitable for large-scale manufacturing for delivery to women in the resource-limited settings of the developing world.

\section{METHODS}

Study animals, procedures, and $\mathrm{pH}$ analysis. In all, 24 normal cycling female Chinese-origin rhesus macaques (Macaca mulatta), aged 3.5-7 years, were used in this study. Animals were housed either at BIOQUAL (Rockville, MD) or at ABL (Advanced Biosciences Laboratories, Kensington, MD). Nonhuman primate housing, care, and treatments were performed in accordance with the standards of the AAALAC (Association for Assessment and Accreditation of Laboratory Animal Care International), the Animal Welfare Act as amended, the Public Health Service Policy on Humane Care and Use of Laboratory Animals, 2002, and the NIH guidelines for Research Involving Recombinant DNA Molecules. In all studies, animals were sedated with $10 \mathrm{mg} \mathrm{kg}^{-1}$ ketamine and $1 \mathrm{mg} \mathrm{kg}^{-1}$ acepromazine when required. Vaginal $\mathrm{pH}$ was monitored using a portable HI 99181 Skin pH Meter Hanna Instrument (Woonsocket, RI). We monitored menses by inserting a clean salinedampened cotton-tipped applicator into the monkey's vagina. The swab was then removed and a recording from 0 to 3 was made $(0=$ no blood, 1 = slight, 2 = moderate, 3 = heavy bleeding). The monkeys were trained for this procedure by giving food as a reward. CVLs were taken by instilling $2 \mathrm{ml}$ phosphate-buffered saline spiked with complete, EDTAfree protease Inhibitor (Roche Applied Science, Indianapolis, IN) into the vagina, flushing it back and forth four times, and then collecting the fluids on ice. Samples were clarified by centrifugation at $820 \mathrm{~g}$ at $+4^{\circ} \mathrm{C}$ and snap frozen on dry ice, before storage at $-80^{\circ} \mathrm{C}$ until analysis. Samples were collected in all phases of the menstrual cycle.

Genetic modifications of Lactobacillus. L. jensenii 1153-1666 used in the study was created at Osel (Mountain View, CA) and is described in the study by Liu et al. ${ }^{24}$ In brief, the nucleotide sequence of the $C V-N$ gene was recoded for optimal Lactobacillus codon usage. In addition, proline at amino acid 51 was changed to glycine (P51G) by site-directed mutagenesis to stabilize $\mathrm{CV}-\mathrm{N}$ in a properly folded monomeric form. 
A 4-amino-acid peptide containing APVT (alanine, proline, valine, and threonine), corresponding to the amino terminus of the mature collagenbinding S-layer protein A (CbsA) protein of L. crispatus, was inserted downstream of the CbsA signal sequence to resemble the native signal peptidase cleavage site. ${ }^{24}$ An expression cassette containing an L. jensenii promoter for the ribosomal protein subunit $\left(P_{r p s U}\right)$ directing expression of APVT-CV-N (P51G) was integrated by homologous recombination in a single copy into the pox 1 site of the genome of $L$. jensenii 1153 . The resolved strain, L. jensenii 1153-1666, was used in the macaque studies described in this paper. $L$. jensenii 1153-1666 when cultured to $\sim 10^{8} \mathrm{c}$. f.u. (colony-forming units), secretes modified CV-N at concentrations of approximately $1.5-2 \mu \mathrm{g} \mathrm{ml}^{-1} .{ }^{24} \mathrm{APVT}-\mathrm{CV}-\mathrm{N}$ has similar antiviral activity against CCR5 and CXCR4 viruses as the original CV-N protein. ${ }^{24}$

Lactobacillus growth conditions, colonization, and sampling. Lactobacilli were cultured using the Mann Rogosa Sharp broth or agar purchased from Difco (Becton Dickinson, Franklin Lakes, NJ). A colonization model using the Chinese rhesus macaque for testing a live Lactobacillus-based microbicide was established before this study. ${ }^{25}$ Lactobacilli were delivered to the macaques in a $3 \mathrm{ml}$ volume with equal volumes of Mann Rogosa Sharp and 2.7\% HEC (Hercules Incorporated, Hopewell, VA). The vaginal microflora of each animal was sampled using the Port-A-Cul swab/tube collection system (Becton Dickinson, Cockeysville, MD). Vaginal swabs were taken 2 days before each challenge and plated to Mann Rogosa Sharp agar (Difco, Becton Dickinson) and PRAS Brucella plates (Anaerobe Systems, Morgan Hill, CA). Lactobacilli were identified using Gram stain, and API Microorganism Identification Strips (Biomerieux, St Louis, MO). Lactobacillus sp. identity was confirmed by DNA extraction and $16 \mathrm{~S}$ ribosomal DNA (rDNA) sequencing and BLASTn DNA sequence analysis.

Detection of CV-N in CVL. Macaques were colonized with live L. jensenii 1153-1666 in HEC without the use of antibiotic to determine the extent and duration of colonization. CVLs were performed to detect secreted $\mathrm{CV}-\mathrm{N}$ protein collected at $24 \mathrm{~h}$, and at 3 and 6 weeks after Lactobacillus inoculation. To detect CV-N, CVL samples were incubated with a rabbit pAb against CV-N (Cocalico Biologicals, Reamstown, PA). The mixture was then coupled to the prewashed and pre-equilibrated proteinA sepharose beads (Sigma-Aldrich, St Louis, MO) at $4^{\circ} \mathrm{C}$ for $4 \mathrm{~h}$ under agitation. The beads were washed with lysis buffer, and immunoprecipitated proteins were separated on 4-12\% SDS-PAGE gel and transferred to polyvinylidene fluoride membranes using an IBlot system (Invitrogen, Carlsbad, CA). CV-N present in the CVL was visualized using a goat anti-CV-N pAb (Cocalico Biologicals) and WesternBreeze Chromogenic kit (Invitrogen). Control CV-N used in western blots did not contain the N-terminal APVT sequence and had a slightly faster mobility on SDS-PAGE gels.

Vaginal biopsies and in situ detection of CV-N. Vaginal pinch biopsies were collected from L. jensenii 1153-1666-colonized macaques and either formalin fixed and stained with hematoxylin and eosin or flash frozen for immunohistochemistry. Samples for immunohistochemistry were microsectioned and reacted with goat anti-CV-N antibody diluted 1:4,000 in phosphate-buffered saline containing $1 \%$ bovine serum albu$\mathrm{min}$. Secondary antibody was anti-goat conjugated with immunoperoxidase diluted 1:500 in phosphate-buffered saline-bovine serum albumin. $\operatorname{DAB}\left(3,3^{\prime}\right.$-diaminobenzidine) substrate (Sigma-Aldrich) was used to visualize the $\mathrm{CV}-\mathrm{N}$ in tissue. All immunohistochemistry procedures were performed by Histoserv (Germantown, MD). The sections were visualized using light microscopy on a Leica SP5 Confocal Microscope (Leica Microsystems, Mannheim, Germany). Slides were viewed and photographed using either a $\times 10$ low-power objective or $\times 63$ NA 1.4 oil immersion objective.

Cytokine and immunoglobulin measurements. The levels of IL-1 $\beta$, IL-1RA, IL-6, and IL-8 in CVLs were determined from 16 of the macaques used in the challenge experiment, with a multiplexed fluorescent microsphere immunoassay using the Luminex 100 system (Luminex, Austin, TX) using Milliplex MAP nonhuman primate cytokine kit (Millipore, Billerica, MA). Data were collected and analyzed using Bioplex Manager v3.0 software (Bio-Rad, Hercules, CA) using a fiveparameter fitting algorithm. To account for possible differences between tests, samples were analyzed in overlapping batches testing all the time points from the individual animals at the same time. $\mathrm{CV}-\mathrm{N}$-specific and L. jensenii-specific IgG, IgA, and IgM from macaque CVL were measured by ELISA. NUNC Maxisorp (Thermo Scientific, Rochester, NY) plates were coated with CV-N protein or L. jensenii 1153 whole bacteria, blocked with $1 \%$ casein (Thermo Scientific), and $10 \%$ heat-inactivated goat serum. CVLs and serum samples were tested for the presence of antibodies to CV-N and Lactobacillus using goat anti-monkey IgA, IgG, and IgM (Rockland Immunochemicals, Gilbertsville, PA) and visualized with $p$-nitrophenyl phosphate, disodium salt (Sigma-Aldrich) substrate at $405 \mathrm{~nm}$. Control macaques were immunized with CV-N delivered in Titermax Gold Adjuvant (Sigma-Aldrich). Immunized macaques reached serum titers against $\mathrm{CV}-\mathrm{N}$ of up to $1: 36,000$ after immunization and antibodies to $\mathrm{CV}-\mathrm{N}$ were detected in CVLs collected after the second immunization.

SHIV stock. For expansion of $\mathrm{SHIV}_{\mathrm{SF} 162 \mathrm{P} 3}$, a naive Indian rhesus macaque, which was shown to be free from SRV, STLV, B and foamy viruses, was infected intravenously with $1 \mathrm{ml}$ of cell-free SHIV $\mathrm{SF}_{\text {162P3 }}$ stock. The infected macaque was monitored for plasma viremia and when the RNA load reached $\sim 10^{8}$ copies per ml, peripheral blood mononuclear cells (PBMCs) were harvested, depleted of CD8 + T cells, and activated with phytohemagglutinin for $72 \mathrm{~h}$. Activated CD $4+$ T-cell-enriched PBMCs were then co-cultured with phytohemagglutinin-activated PBMCs pooled from three macaques. Culture media were replenished as required with fresh medium every $2-3$ days and fresh phytohemagglutinin-activated rhesus PBMCs were added after 5 days. The culture was monitored for the presence of SIV Gag p27 protein using antigen capture ELISA kit (ABL) and when the SIV p27 level reached $>50 \mathrm{ng} \mathrm{ml}^{-1}$, cellfree supernatant was harvested, filtered, aliquoted into $1.0 \mathrm{ml}$ aliquots, and stored in the vapor phase of liquid nitrogen.

MatTek culture. The MatTek EpiVaginal Tissue Model VLC-100FT (MatTek) was used to assess the effect of $L$. jensenii $1153-1666$ vs. L. jensenii 1153 (parental strain) on HIV-1 replication. EpiVaginal tissue VLC-100 FT, which includes vaginal-ectocervical epithelial cells, fibroblast-containing lamina propria, and dendritic cells, was infected with HIV- $1_{\mathrm{SF} 162}$ at a p24 concentration of $3 \mathrm{ng} \mathrm{ml}^{-1}$ in $100 \mu \mathrm{l}$, in the presence of $10^{7}$ c.f.u. L. jensenii $1153-1666$ or $10^{7}$ c.f.u. L. jensenii 1153 (parental strain). Two assays were performed with duplicate tissue replicates for each sample. Samples of tissue culture media were harvested for $\mathrm{p} 24$ analysis at day 1, 3, 5, and 8 after infection. HIV-1 p24 concentrations were determined using ELISA (Perkin-Elmer, Waltham, MA).

SHIV challenge study. In the SHIV challenge study, azithromycin suppositories were administered vaginally to 12 macaques to clear their Lactobacillus flora. After a 1-week rest, the 12 macaques in the experimental arm were colonized with L. jensenii 1153-1666 in $\sim 3 \mathrm{ml}$ of a 1:1 ratio of Mann Rogosa Sharp broth and HEC daily five times in the first week and 2 times each after week with SHIV challenge 24-48 h later. Control animals $(n=12)$ received the following: azithromycin treatment (as described above), followed by $2.7 \%$ HEC (as described) $(n=4), 2.7 \%$ HEC (as described) $(n=4)$, or no treatment $(n=4)$.

SHIV challenges were performed 24-48 h after Lactobacillus or HEC inoculation. In all, $1 \mathrm{ml}$ of RPMI containing $300 \mathrm{TCID}_{50} \mathrm{SHIV}_{\mathrm{SF} 162 \mathrm{P} 3}$ in RMPI was intravaginally inoculated using a $3-\mathrm{cm}^{3}$ syringe in an atraumatic manner. Animals were challenged up to six times or until infection was established by a positive plasma viral load.

Viral load determination and seroconversion. Quantitative SHIV reverse transcriptase-PCR was performed as previously described. ${ }^{49}$ Protection was defined as absence of plasma viremia (RNA viral load) at weekly time points from 7 to 70 days after challenge. Infection was 
defined by consecutive positive viral RNA detections $>50$ copies SHIV RNA per ml. Plasma samples obtained from all macaques were tested for seroconversion and antibody titers to $\mathrm{p} 27$, a SIV core antigen, using an ELISA (performed at ABL). In addition, all plasma samples were tested by a western blot assay (ZeptoMetrix, Buffalo, NY) to detect antibodies to SIV antigens using a modified procedure recommended by the manufacturer.

Trim5 $\alpha$ allele testing. TaqMan reverse transcriptase-PCR was used to amplify Trim $5 \alpha$ alleles from PBMCs in 15 macaques (BIOQUAL). In five macaques, genomic sequence was determined from blood (Harvard NEPRC, Southborough, MA). Four macaques were not tested because of insufficient numbers of PBMCs available.

Statistical analysis. Paired Student's $t$-tests were used to analyze the vaginal $\mathrm{pH}$ data. The cytokine data and HIV-1 inhibition in MatTek cultures were analyzed with nonparametric Wilcoxon's matched-pair signed-rank test using Prism Version 5 (GraphPad Software, La Jolla, CA). The proportion of uninfected vs. infected animals was analyzed using Kaplan-Meier survival analysis, and peak viral loads compared using the Student's $t$ test, in JMP Version 8 (SAS, Cary, NC). All hypothesis tests were two-tailed, and a $P$-value of $\leqslant 0.05$ was considered statistically significant.

SUPPLEMENTARY MATERIAL is linked to the online version of the paper at http://www.nature.com/mi

\section{ACKNOWLEDGMENTS}

The SHIV virus stock was produced under NIAID contract HHSN272200800020C by ABL. NIH's Intramural AIDS Targeted Antiviral Program (IATAP) provided funding for the challenge study. This work was also supported by NIH grant 5R33AI071978-04. We thank Laurent Pessaint for preparation of Lactobacillus, placebo, and animal blood processing, Jack Greenhouse for quantitative SHIV RT-PCR, Matt Collins and Jermaine Hoes for animal sampling and care. We thank Jennifer Morgan and the Welkin E Johnson Laboratory (Harvard Medical School) for performing TRIM5 $\alpha$ sequencing. We thank Owen Schwartz and the Biological Imaging Facility for assistance with microscopy. We also thank Mario Roederer for critical reading of the manuscript.

\section{DISCLOSURE}

LAL, XL, YL, and PPL work for Osel, Mountain View, CA, a company developing this approach as a topical microbicide. BIOQUAL (BES) and $\mathrm{ABL}(\mathrm{RP})$ are for-profit institutions and are subawardees to Osel.

(C) 2011 Society for Mucosal Immunology

\section{REFERENCES}

1. Sabin, L.L. et al. Barriers to adherence to antiretroviral medications among patients living with HIV in southern China: a qualitative study. AIDS Care 20, 1242-1250 (2008).

2. Hillier, S.L. et al. In vitro and in vivo: the story of nonoxynol 9. J. Acquir. Immune. Defic. Syndr. 39, 1-8 (2005).

3. Abdool Karim, S.S. Results of effectiveness trials of PRO 2000 gel: lessons for future microbicide trials. Future Microbiol. 5, 527-529 (2010).

4. Van Damme, L. et al. Lack of effectiveness of cellulose sulfate gel for the prevention of vaginal HIV transmission. N. Engl. J. Med. 359, 463-472 (2008).

5. Skoler-Karpoff, S. et al. Efficacy of Carraguard for prevention of HIV infection in women in South Africa: a randomised, double-blind, placebocontrolled trial. Lancet 372, 1977-1987 (2008).

6. Roddy, R.E. et al. A controlled trial of nonoxynol 9 film to reduce male-tofemale transmission of sexually transmitted diseases. N. Engl. J. Med. 339, 504-510 (1998).

7. Grant, R.M. et al. Whither or wither microbicides? Science 321, 532-534 (2008).

8. Cohen, J. AIDS research. Microbicide fails to protect against HIV. Science 319, 1026-1027 (2008).
9. Karim, Q.A. et al. Effectiveness and safety of Tenofovir Gel, an antiretroviral microbicide, for the prevention of HIV infection in women. Science 329, 1168-1174 (2010).

10. Haase, A.T Targeting early infection to prevent HIV-1 mucosal transmission. Nature 464, 217-223 (2010).

11. Hladik, F. \& Hope, T.J. HIV infection of the genital mucosa in women. Curr. HIVIAIDS Rep. 6, 20-28 (2009).

12. Hladik, F. et al. Initial events in establishing vaginal entry and infection by human immunodeficiency virus type-1. Immunity 26, 257-270 (2007).

13. Hillier, S.L. The vaginal microbial ecosystem and resistance to HIV. AIDS Res. Hum. Retroviruses 14 (Suppl 1), S17-S21 (1998).

14. Boskey, E.R., Cone, R.A., Whaley, K.J. \& Moench, T.R. Origins of vaginal acidity: high $\mathrm{D} / \mathrm{L}$ lactate ratio is consistent with bacteria being the primary source. Hum. Reprod. 16, 1809-1813 (2001).

15. Hawes, S.E. et al. Hydrogen peroxide-producing lactobacilli and acquisition of vaginal infections. J. Infect. Dis. 174, 1058-1063 (1996).

16. Martin, H.L. et al. Vaginal lactobacilli, microbial flora, and risk of human immunodeficiency virus type 1 and sexually transmitted disease acquisition. J. Infect. Dis. 180, 1863-1868 (1999).

17. Chang, T.L. et al. Inhibition of HIV infectivity by a natural human isolate of Lactobacillus jensenii engineered to express functional two-domain CD4. Proc. Natl Acad. Sci. USA 100, 11672-11677 (2003).

18. Boyd, M.R. et al. Discovery of cyanovirin-N, a novel human immunodeficiency virus-inactivating protein that binds viral surface envelope glycoprotein gp120: potential applications to microbicide development. Antimicrob. Agents Chemother. 41, 1521-1530 (1997).

19. Dey, B. et al. Multiple antiviral activities of cyanovirin-N: blocking of human immunodeficiency virus type $1 \mathrm{gp} 120$ interaction with CD4 and coreceptor and inhibition of diverse enveloped viruses. J. Virol. 74, 4562-4569 (2000).

20. Buffa, V. et al. Cyanovirin-N potently inhibits human immunodeficiency virus type 1 infection in cellular and cervical explant models. J. Gen. Virol. $90,234-243$ (2009).

21. Alexandre, K.B. et al. Mannose-rich glycosylation patterns on HIV-1 subtype C gp120 and sensitivity to the lectins, Griffithsin, Cyanovirin-N and Scytovirin. Virology 402, 187-196 (2010).

22. Tsai, C.C. et al. Cyanovirin-N inhibits AIDS virus infections in vaginal transmission models. AIDS Res. Hum. Retroviruses 20, 11-18 (2004).

23. Tsai, C.C. et al. Cyanovirin- $\mathrm{N}$ gel as a topical microbicide prevents rectal transmission of SHIV89.6P in macaques. AIDS Res. Hum. Retroviruses 19, 535-541 (2003).

24. Liu, X. et al. Engineered vaginal Lactobacillus strain for mucosal delivery of the human immunodeficiency virus inhibitor cyanovirin-N. Antimicrob. Agents Chemother. 50, 3250-3259 (2006).

25. Yu, R.R. et al. A Chinese rhesus macaque (Macaca mulatta) model for vaginal Lactobacillus colonization and live microbicide development. J. Med. Primatol. 38, 125-136 (2009).

26. Eschenbach, D.A. et al. Influence of the normal menstrual cycle on vaginal tissue, discharge, and microflora. Clin. Infect. Dis. 30, 901-907 (2000).

27. Spear, G.T. et al. Identification of rhesus macaque genital microbiota by $16 S$ pyrosequencing shows similarities to human bacterial vaginosis: implications for use as an animal model for HIV vaginal infection. AIDS Res. Hum. Retroviruses 26, 193-200 (2010).

28. Fichorova, R.N. Guiding the vaginal microbicide trials with biomarkers of inflammation. J. Acquir. Immune. Defic. Syndr. 37 (Suppl 3), S184-S193 (2004).

29. Trifonova, R.T. et al. Biomarkers of leukocyte traffic and activation in the vaginal mucosa. Biomarkers 12, 608-622 (2007).

30. Atashili, J., Poole, C., Ndumbe, P.M., Adimora, A.A. \& Smith, J.S. Bacterial vaginosis and HIV acquisition: a meta-analysis of published studies. Aids 22, 1493-1501 (2008).

31. Denenberg, R. HIV and the vaginal ecosystem. GMHC Treat Issues 11, 8-10 (1997).

32. McClelland, R.S. et al. Improvement of vaginal health for Kenyan women at risk for acquisition of human immunodeficiency virus type 1: results of a randomized trial. J. Infect. Dis. 197, 1361-1368 (2008).

33. Fox, C.A., Meldrum, S.J. \& Watson, B.W. Continuous measurement by radio-telemetry of vaginal $\mathrm{pH}$ during human coitus. J. Reprod. Fertil. 33, 69-75 (1973).

34. Hemmerling, A. et al. Phase 2a study assessing colonization efficiency, safety, and acceptability of Lactobacillus crispatus CTV-05 in women with bacterial vaginosis. Sex Transm. Dis. 37, 745-750 (2010). 


\section{ARTICLES}

35. Huskens, D., Vermeire, K., Vandemeulebroucke, E., Balzarini, J. \& Schols, D. Safety concerns for the potential use of cyanovirin-N as a microbicidal anti-HIV agent. Int. J. Biochem. Cell. Biol. 40, 2802-2814 (2008).

36. Mori, T. et al. Functional homologs of cyanovirin-N amenable to mass production in prokaryotic and eukaryotic hosts. Protein Expr. Purif. 26, 42-49 (2002).

37. Keele, B.F. et al. Identification and characterization of transmitted and early founder virus envelopes in primary HIV-1 infection. Proc. Natl Acad. Sci. USA 105, 7552-7557 (2008).

38. Keele, B.F. et al. Low-dose rectal inoculation of rhesus macaques by SIVsmE660 or SIVmac251 recapitulates human mucosal infection by HIV-1. J. Exp. Med. 206, 1117-1134 (2009).

39. Hudgens, M.G. et al. Power to detect the effects of HIV vaccination in repeated low-dose challenge experiments. J. Infect. Dis. 200, 609-613 (2009).

40. Lederman, M.M. et al. Prevention of vaginal SHIV transmission in rhesus macaques through inhibition of CCR5. Science 306, 485-487 (2004).

41. Veazey, R.S. et al. Protection of macaques from vaginal SHIV challenge by vaginally delivered inhibitors of virus-cell fusion. Nature 438, 99-102 (2005).

42. Shattock, R.J. \& Moore, J.P. Inhibiting sexual transmission of HIV-1 infection. Nat. Rev. Microbiol. 1, 25-34 (2003).

43. Haase, A.T. Early events in sexual transmission of HIV and SIV and opportunities for interventions. Annu. Rev. Med. 62, 127-139 (2011).
44. Vogel, T., Norley, S., Beer, B. \& Kurth, R. Rapid screening for Mamu-A1positive rhesus macaques using a SIVmac Gag peptide-specific cytotoxic T-lymphocyte assay. Immunology 84, 482-487 (1995).

45. Wang, W. et al. Frequency of the major histocompatibility complex Mamu$\mathrm{A}^{*} 01$ allele in experimental rhesus macaques in China. J. Med. Primatol. 39, 374-380 (2010).

46. Amirfar, S., Hollenberg, J.P. \& Abdool Karim, S.S. Modeling the impact of a partially effective HIV vaccine on HIV infection and death among women and infants in South Africa. J. Acquir. Immune. Defic. Syndr. 43, 219-225 (2006).

47. Masse, B.R., Boily, M.C. \& Desai, K. Using mathematical modeling to bridge phase 3 microbicide trials with public health decision making. J. Acquir. Immune. Defic. Syndr. 50, 434-435 (2009).

48. Vangelista, L. et al. Engineering of Lactobacillus jensenii to secrete RANTES and a CCR5 antagonist analogue as live HIV-1 blockers. Antimicrob. Agents Chemother. 54, 2994-3001 (2010).

49. Lewis, M.G. et al. Response of a simian immunodeficiency virus (SIVmac251) to raltegravir: a basis for a new treatment for simian AIDS and an animal model for studying lentiviral persistence during antiretroviral therapy. Retrovirology 7, 21 (2010).

(c) This work is licensed under the Creative Commons

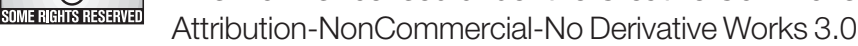

Unported License. To view a copy of this license, visit http://creativecommons.org/licenses/by-nc-nd/3.0/ 